\title{
Estimativas de parâmetros genéticos e fenotípicos de características do pelame e de desempenho reprodutivo de vacas holandesas em clima tropical ${ }^{1}$
}

\author{
Elaine Cristina Abaker Bertipaglia ${ }^{2}$, Roberto Gomes da Silva ${ }^{3}$, Vania Cardoso ${ }^{4}$, \\ Alex Sandro Campos Maia ${ }^{5}$
}

\footnotetext{
1 Parte da dissertação de Mestrado da primeira autora (FCAV - UNESP, Jaboticabal).

${ }^{2}$ Doutoranda em Medicina Veterinária (área de concentração: Reprodução Animal) - FCAV - UNESP - Jaboticabal, CEP: 14884-900, Jaboticabal - SP.

${ }^{3}$ Laboratório de Bioclimatologia, Departamento de Zootecnia, FCAV - UNESP - Jaboticabal - SP.

4 Doutora em Zootecnia, Gensys consultores associados S/S LTDA.

5 Doutor em Zootecnia.
}

RESUMO - Objetivou-se investigar os efeitos das características do pelame sobre a idade ao primeiro parto (IPP) e o intervalo de partos (IEP) de vacas holandesas manejadas em sistema de estabulação livre com ventiladores e aspersores e estimar os parâmetros genéticos destas características. Os dados foram analisados pelo método dos quadrados mínimos, considerando os efeitos: ano; estação; número de inseminações; origem do pai; pai dentro da origem; idade (somente para IEP); porcentagem de malhas negras; espessura do pelame; comprimento dos pêlos; número de pêlos por unidade de área da epiderme; diâmetro dos pêlos; transmitância e refletância efetiva do pelame. O método da Máxima Verossimilhança Restrita foi utilizado para estimar os componentes de (co)variância sob um modelo animal. Os resultados incluíram as estimativas de herdabilidade para IPP $(0,23 \pm 0,08)$, IEP $(0,19 \pm 0,10)$, malhas negras $(0,75 \pm 0,08)$, número $(0,05 \pm 0,04)$, espessura $(0,04 \pm 0,05)$, comprimento $(0,36 \pm 0,09)$ e diâmetro $(0,63 \pm 0,08)$ de pêlos. As estimativas de correlação genética entre IPP $(-0,37 \pm 0,17), \operatorname{IEP}(0,49 \pm 0,27)$ e diâmetro apresentaram valores significativos e favoráveis. Entretanto, as correlações genéticas entre espessura $(-0,56 \pm 0,46)$, número $(-0,66 \pm 0,43)$, porcentagem de malhas negras $(0,04 \pm 0,16)$ e IPP foram desfavoráveis para seleção conjunta para melhor adaptação e precocidade sexual. O alto valor estimado para herdabilidade e as correlações genéticas favoráveis entre diâmetro e IPP e IEP indicaram ser possível selecionar para melhorar conjuntamente a adaptação e o desempenho reprodutivo.

Palavras-chave: adaptação, bovinos, correlações genéticas, herdabilidade, idade ao primeiro parto, intervalo de partos

\section{Genetic and phenotypic parameters for hair coat and reproduction traits of Holstein cows in tropical environment}

\begin{abstract}
The present study aimed to evaluate the effects of hair coat on age at first calving (IPP) and calving interval (IEP) of Holstein cows managed under free stall with fans and sprinklers and to estimate genetic and phenotypic parameters for these traits. Data were analyzed by the least-squares method using a model that included the effects of year; season; origin of sire; sire within origin; number of inseminations; age (only for calving interval); percentage of black meshes; hair coat thickness; hair length; number of hair per unit area; hair diameter; effective transmissivity and reflectivity. Covariance components were estimated by REML using an Animal Model. Heritability estimates for IPP, IEP, percentage of black meshes, number of hair per unit area, hair coat thickness, hair length and hair diameter were $0.23 \pm 0.08,0.19 \pm 0.10,0.75 \pm 0.08$, $0.05 \pm 0.04,0.04 \pm 0.05,0.36 \pm 0.09$, and $0.63 \pm 0.08$, respectively. Genetic correlation between hair diameter and IPP (IEP) was equal to $-0.37 \pm 0.17(-0.49 \pm 0.27)$. However, genetic correlations between IPP and hair thickness $(-0.56 \pm 0.46)$, number of hair $(-0.66 \pm 0.43)$ and black meshes $(0.04 \pm 0.16)$ were unfavorable for jointly selection for sexual precocity and fitness to tropical environment. High values of heritability estimates and genetic correlations between diameter, IPP and IEP indicate that is possible to select for thickness of hair diameter and improve reproductive efficiency.
\end{abstract}

Key Words: age at first calving, bovine, calving interval, fitness, genetic correlation, heritability

\section{Introdução}

Os animais em geral são adaptáveis, capazes de manter a vida, os desempenhos produtivo e reprodutivo em uma ampla gama de ambientes físicos, químicos, biológicos e sociais. Entretanto, condições térmicas adversas ocorrem no ambiente normal dos animais, causando-lhes estresse e levando-os a reduções no desempenho, como resultado da 
diminuição na saúde e na higidez. Dentro de certos limites, os animais podem se ajustar fisiológica, comportamental ou imunologicamente de modo a sustentar a homeostase orgânica e minimizar as conseqüências adversas. Neste processo de ajuste, entretanto, as funções menos vitais ao organismo, como o desempenho (produção e reprodução) e o bemestar, podem ser atingidas quando a intensidade e a duração dos estressores ambientais excedem a capacidade compensatória dos animais, geneticamente determinada.

A função reprodutiva é particularmente sensível aos fatores de estresse, especialmente o estresse térmico. Conseqüentemente, temperatura e umidade elevadas causam períodos transientes de infertilidade nos animais (Thatcher, 1974; Wolfenson et al., 2000; Rivera \& Hansen, 2001). Há evidências ainda de que a hipertermia decorrente de problemas adaptativos afeta adversamente o desenvolvimento dos embriões, causando maior incidência de mortes embrionárias (Putney et al., 1989). A redução sazonal da eficiência reprodutiva em gado leiteiro é um dos mais sérios problemas nas áreas intertropicais do mundo (Gwazdauskas et al., 1975; Badinga et al., 1985) e as taxas de concepção diminuem de 40 a $60 \%$ nos meses frios para 10 a $20 \%$ ou menos no verão, dependendo da severidade do estresse térmico (Cavestany et al, 1985).

Além dos fatores ambientais que interferem no equilíbrio térmico dos animais, o pelame é essencial nas trocas térmicas organismo versusambiente. A estrutura física de suas fibras e a camada de ar armazenada no pelame promove misolamento térmico e proteção contra a radiação solar direta (Silva, 2000a; Silva et al., 2001). O pelame está diretamente relacionado à capacidade do animal em perder ou ganhar calor do ambiente, por isso, os diversos tipos de pelames envolvem isolamento térmico, eficiência de termólise evaporativa, atributos termorreguladores (entre tipo de pelame e sudação) e associação do tipo de pelame com produção, ganho de peso, reprodução e outras características não ligadas diretamente à termorregulação (Turner, 1964).

O gado da raça Holandesa, desenvolvido em ambientes nos quais o estresse por calor é mínimo, apresenta, em geral, características pouco adequadas à dissipação do excesso de calor corporal decorrente do elevado desempenho produtivo, fato agravado pelas altas temperaturas e pela forte carga térmica radiante do ambiente tropical brasileiro. A queda sazonal na produção e reprodução de vacas Holandesas de alta produção coloca em perspectiva a importância das diferenças no tipo de pelame de uma raça de origem de clima temperado em um ambiente tropical úmido. Diferenças na adaptação entre membros de um grupo assumem importância significativa à medida que a discrepância entre ambiente e nível médio de adaptação do grupo aumenta e, conseqüentemente, resultam em baixo desempenho (Hansen, 1997).

Apesar da importância econômica do rebanho holandês no Brasil e, não obstante o grande número de trabalhos realizados em outros países, poucos estudos têm sido realizados para analisar as conseqüências das características adaptativas do pelame sobre os desempenhos produtivo e reprodutivo desses animais.

Este trabalho foi realizado com o objetivo de investigar os efeitos das características do pelame sobre a idade ao primeiro parto e o intervalo de partos de vacas Holandesas mantidas sob manejo, nutrição e sanidade otimizados. Estimaram-se ainda os parâmetros genéticos destas características (herdabilidade e correlações), visando ao melhoramento genético para adaptação desta raça às condições de clima tropical.

\section{Material e Métodos}

Os dados utilizados neste trabalho foram provenientes de animais pertencentes ao rebanho da Fazenda Santa Rita, Agrindus S.A., localizado no município de Descalvado, São

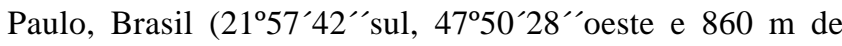
altitude). Foram avaliadas 918 vacas holandesas de 2 a 13 anos de idade, filhas de 199 touros (21 brasileiros, 38 canadenses e 140 americanos). Os animais eram manejados em sistema intensivo em galpões tipo free-stall, providos de sistema de resfriamento por ventiladores e aspersores. A média de produção dos animais era de $30 \mathrm{~kg} /$ vaca/dia, em três ordenhas diárias. As novilhas eram inseminadas ao atingirem $350 \mathrm{~kg}$ de $\mathrm{PV}$, geralmente com 12-13 meses de idade. A detecção de cios era realizada por observação visual durante a madrugada, sempre por uma única pessoa. A inseminação era feita 60 dias após o parto ou no segundo cio com muco limpo. O diagnóstico de gestação era realizado por palpação retal a partir dos 45 dias após a inseminação.

Os dados reprodutivos de anos sucessivos foram obtidos a partir das fichas individuais do rebanho, contendo número de identificação do animal, data de nascimento, identificação do pai e da mãe, país de origem do pai, datas das inseminações com gestação confirmada, datas dos partos, ordem de parição e número de inseminações por parto. Foram avaliados a idade ao primeiro parto (IPP) e o intervalo de partos (IEP). Somente os desempenhos reprodutivos, da primeira e da segunda parição, foram considerados. Animais com dados incompletos foram 
des cartados. Ao final desse processo seletivo, permaneceram 918 animais com um parto e, entre esses, 595 com dois partos.

Foram determinadas as seguintes características do pelame: proporção de área de malhas negras em relação à superfície corporal (P, \%); espessura da capa de pelame (E, $\mathrm{mm})$ comprimento médio dos pêlos $(\mathrm{C}, \mathrm{mm})$; número de pêlos por unidade de área da epiderme $\left(\mathrm{N}\right.$, pêlos $\left./ \mathrm{cm}^{2}\right)$; e diâmetro médio dos pêlos $(\mathrm{D}, \mu \mathrm{m})$. À exceção da proporção de área de malhas negras em relação à superfície corporal, todas as características morfológicas foram avaliadas tanto para o pelame preto como para o branco em cada animal, de acordo com Silva (2000a), sendo consideradas média ponderada em relação à proporção de malhas negras e brancas.

As medidas do pelame e a coleta de amostras de pêlos dos animais foram realizadas no período de novembro de 2000 a abril de 2001. Os pêlos foram retirados aproximadamente $20 \mathrm{~cm}$ abaixo da coluna vertebral, na região torácica mediana. No caso de animais com malhas preta e branca, foram retiradas nesta região amostras separadas para cada cor de malha utilizando-se o método proposto por Silva (2000a), por meio de um alicate comum de eletricista com o bico limado, para facilitar a penetração no pelame no momento da coleta. Os pêlos foram arrancados de uma área total de $18 \mathrm{~mm}^{2}$ da pele do animal, acondicionados em envelopes plásticos e identificados.

A proporção relativa de malha negra em relação à área corporal foi determinada por avaliação visual direta em ambos os lados de cada animal, exceto cauda, membros e a região ventral do tronco, de acordo com Becerril \& Wilcox (1992). A espessura da capa de pelame foi determinada diretamente nos animais, no mesmo local onde foi retirada a amostra de pêlos, utilizando-se um paquímetro, introduzido perpendicularmente à superfície do pelame até tocar suavemente a pele. O cursor era então movido até tocar a superfície externa do pelame, efetuando-se a leitura em milímetros (Silva, 2000a).

Para determinação do comprimento médio dos pêlos, foi realizada a média aritmética do comprimento dos dez maiores pêlos, eleitos pela análise visual da amostra e medidos com paquímetro. Os pêlos das amostras foram espalhados em uma placa de vidro colocada sobre fundo contrastante, alinhando-se com uma pinça e efetuando a medição dos dez maiores pêlos. O diâmetro médio dos pêlos foi medido utilizando-se um micrômetro e obtido pela média aritmética do diâmetro dos dez maiores pêlos. A densidade numérica do pelame foi estimada pela contagem do número total de pêlos da amostra, correspondente à área de $18 \mathrm{~mm}^{2}$ de pele do animal. A contagem foi realizada com auxílio de um contador manual e de uma pinça. Determinada a quanti- dade de pêlos da amostra, procedeu-se à conversão para número de pêlos por centímetro quadrado (Silva, 2000a).

As propriedades radiativas efetivas do pelame foram definidas por Cena \& Monteith (1975), tanto para áreas negras como para as brancas. A refletância $\left(\rho^{*}\right)$ e transmitância $\left(\tau^{*}\right)$ foram calculadas de acordo com as seguintes equações:

$\rho^{*}=\frac{\left[\rho_{c}\left(1-\rho_{s}\right)-\alpha_{c} \rho_{s}\right] \operatorname{senh} x+k \rho_{s} \cosh x}{\left[\rho_{c}\left(1-\rho_{s}\right)+\alpha_{c}\right] \operatorname{senh} x+k \cosh x}$

$\tau^{*}=\frac{k}{\left[\rho_{c}\left(1-\rho_{s}\right)+\alpha_{c}\right] \operatorname{senh} x+k \cosh x}$

$k=\left[\alpha_{c}^{2}+2 \rho_{c} \alpha c\right]^{0,5}$

$x=K E P$

$P=N D\{\tan [\arccos (E / C)]\}$

em que: senhx e $\cosh \mathrm{x}=$ seno e cosseno hiperbólicos de $\mathrm{x}$, respectivamente; $\mathrm{P}=$ fração da energia radiante interceptada pela unidade de espessura da capa de pelame; $\mathrm{N}=$ número de pêlos (pêlos $\left./ \mathrm{m}^{2}\right) ; \mathrm{D}=$ diâmetro médio $(\mathrm{m}) ; \mathrm{E}=$ espessura (m); e $\mathrm{C}=$ comprimento médio dos pêlos $(\mathrm{m})$. As propriedades radiativas da superfície da pele $\rho_{\mathrm{s}}$ (refletância da superfície da pele) e do pelame $\rho_{\mathrm{c}}$ (refletância da superfície do pelame); e $\alpha_{c}$ (absortância da superfície do pelame) corresponderam às médias das medidas feitas com amostras de pele frescas obtidas de animais mortos provenientes do laboratório de Patologia Animal da UNESP, Campus de Jaboticabal. As determinações foram feitas em um espectroradiômetro, modelo Li-Cor LI-1800 para comprimento de ondas de 300-850 nm. As amostras foram obtidas da mesma área do corpo onde foram retiradas as amostras de pêlos. Os valores estimados foram: $\rho_{\mathrm{c}}($ negro $)=0,0893 ; \rho_{\mathrm{c}}($ branco $)=$ 0,$4526 ; \tau_{\mathrm{s}}($ negro $)=0,0713 ; \tau_{\mathrm{s}}($ branco $)=0,3920 ; \alpha_{\mathrm{c}}$ (negro) $=$ 0,$9020 ; \alpha_{\mathrm{c}}($ branco $)=0,5180$.

Os dados foram inicialmente analisados pelo método dos quadrados mínimos (Littell et al., 1991). A distribuição dos erros da variável idade ao primeiro parto obedeceu à normalidade. Todavia, a variável intervalo de partos foi transformada, pois os erros não obedeceram a uma distribuição normal. A seguinte equação foi adotada na transformação, segundo o método de Box \& Cox (1964):

$y^{\prime}=\frac{y^{\lambda}-1}{\lambda}$

em que $\lambda=0,10$.

$O$ valor de $\lambda$ foi estimado utilizando-se o método da Máxima Verossimilhança, conforme Drapper \& Smith (1998). O modelo matemático utilizado na análise da idade ao primeiro parto foi descrito como:

$\mathrm{Y}_{i j k l m n o p q r s}=\alpha+\mathrm{A}_{i}+\mathrm{M}_{j}+\mathrm{I}_{k}+\mathrm{P}_{l}+\mathrm{E}_{m}+\mathrm{C}_{n}+\mathrm{D}_{o}+\mathrm{N}_{p}+\mathrm{O}_{q}$ $+\mathrm{S}_{q r}+\mathrm{b}_{1}\left(\tau_{1}{ }^{*} \mathrm{~T}\right)+\mathrm{b}_{2}\left(\tau_{2}{ }^{*} \mathrm{~T}\right)+\mathrm{b}_{3}\left(\tau_{3}{ }^{*} \mathrm{~T}\right)+\mathrm{b}_{4}\left(\rho^{*}-\mathrm{R}\right)+$ 
$\mathrm{b}_{5}\left(\rho^{*-} \mathrm{R}\right)+\mathrm{b}_{6}\left(\rho^{*-} \mathrm{R}\right)+\mathrm{e}_{i j k l m n o p q r s}$

em que: $Y_{i j k l m n o p q r s}=$ média da idade ao primeiro parto da $s$-ésima vaca; $\mathrm{A}_{i}=$ efeito fixo do ano do nascimento ( $i=1, \ldots, 12 ;$ de 1990 a 2001); $\mathrm{M}_{j}=$ efeito fixo daj-ésima classe de estação do ano do nascimento $(j=1, \ldots, 4$, sendo $1=$ dezembro a fevereiro; 2 = março a junho; $3=$ julho a setembro e $4=$ outubro a novembro); $\mathrm{I}_{k}=$ efeito fixo do número de inseminações por concepção $(k=1, \ldots, 11) ; \mathrm{P}_{l}=$ efeito fixo de classe da proporção de malhas negras $(l=1, \ldots, 4$; $1=0-25 \% ; 2=26-50 \% ; 3=51-75 \% ; 4=76-100 \%) ; \mathrm{E}_{m}=$ efeito fixo de classe da espessura do pelame $(m=1, \ldots, 4$; sendo $1=\langle 2 \mathrm{~mm} ; 2=2 \geq m \leq 2,5 \mathrm{~mm} ; 3=2,5>m \leq 3 \mathrm{~mm} ; 4=>3 \mathrm{~mm}$ ); $\mathrm{C}_{n}=$ efeito fixo de classe do comprimento dos pêlos $(n=1, \ldots, 4$; sendo $1=<10 \mathrm{~mm} ; 2=10 \geq n \leq 15 \mathrm{~mm}$; $3=15>n \leq 20 \mathrm{~mm} ; 4=>20 \mathrm{~mm}) ; \mathrm{D}_{o}=$ efeito fixo de classe do diâmetro dos pêlos $(o=1, \ldots, 4$; sendo $1=<60 \mu \mathrm{m}$; $2=60 \geq 0 \leq 65 \mu \mathrm{m} ; 3=65>o \leq 70 \mu \mathrm{m} ; 4=>70 \mu \mathrm{m}) ; \mathrm{N}_{p}=$ efeito fixo de classe do número de pêlos por unidade de área $\left(p=1, \ldots, 6\right.$; sendo $1=0-800$ pêlos $/ \mathrm{cm}^{2} ; 2=801-1.200$ pêlos $/ \mathrm{cm}^{2} ; 3=1.201-1.600$ pêlos $/ \mathrm{cm}^{2} ; 4=1.601-2.000$ pêlos $/ \mathrm{cm}^{2} ; 5=2.001-2.500$ pêlos $/ \mathrm{cm}^{2} ; 6=>2.500$ pêlos $/$ $\left(\mathrm{cm}^{2}\right) ; \mathrm{O}_{q}=$ efeito fixo de classe da origem do pai da vaca $(q=1, \ldots, 3 ;$ sendo 1 = Canadá; 2 = Estados Unidos; $3=$ Brasil); $\mathrm{S}_{q r}=$ efeito aleatório do pai da vaca dentro da origem $(r=1, \ldots, 199) ; \mathrm{b}_{1}, \mathrm{~b}_{2}$ e $\mathrm{b}_{3}=$ coeficientes de regressão sobre a transmitância efetiva $\left(\tau^{*}\right) ; b_{4}, b_{5}$ e $b_{6}=$ coeficientes de regressão sobre a refletância efetiva $\left(\rho^{*}\right)$ do pelame para cada comprimento de onda 300-420 nm (1), 420-780 nm (2) e 780-850 nm (3); $\varepsilon_{i j k l m n o p q r s}=$ erro aleatório; e $\alpha=$ intercepto, associado a cada observação.

O modelo utilizado para a análise do intervalo de partos foi o seguinte:

$\mathrm{Y}_{i j k l m n o p q r s t}=\alpha+\mathrm{A}_{i}+\mathrm{M}_{j}+\mathrm{I}_{k}+\mathrm{P}_{l}+\mathrm{E}_{m}+\mathrm{C}_{n}+\mathrm{D}_{o}+\mathrm{N}_{p}+\mathrm{O}_{q}$ $+\mathrm{S}_{q r}+\mathrm{J}_{s}+\mathrm{b}_{1}\left(\tau_{1}{ }^{*}-\mathrm{T}\right)+\mathrm{b}_{2}\left(\tau_{2}{ }^{*}-\mathrm{T}\right)+\mathrm{b}_{3}\left(\tau_{3}{ }^{*} \mathrm{~T}\right)+\mathrm{b}_{4}\left(\rho^{*}-\mathrm{R}\right)$ $+\mathrm{b}_{5}\left(\rho^{*}-\mathrm{R}\right)+\mathrm{b}_{6}\left(\rho^{*}-\mathrm{R}\right)+\varepsilon_{i j k l m n o p q r s t}$

em que: $\mathrm{Y}_{i j k l m n o p q r s t}=$ intervalo de partos dat-ésima vaca; $\mathrm{A}_{i}=$ efeito do ano do primeiro parto $(i=1, \ldots, 11) ; \mathrm{M}_{j}=$ efeito de classe da estação do primeiro parto $(j=1, \ldots, 4)$; $\mathrm{I}_{k}=$ número de inseminações por concepção para o segundo parto $(l=1, \ldots, 17) ; \mathrm{J}_{S}=$ efeito linear da idade ao primeiro parto; os demais termos são como descrito no modelo anterior. As médias gerais para idade ao primeiro parto e para intervalo de partos foram estimadas como:

$\hat{\mu}=\hat{\alpha}+\hat{b}_{1} \bar{T}_{1}+\hat{b}_{2} \overline{T_{2}}+\hat{b}_{3} \bar{T}_{3}+\hat{b}_{4} \bar{R}_{1}+\hat{b}_{5} \bar{R}_{2}+\hat{b}_{6} \overline{R_{3}}$

em que $\alpha=$ intercepto da $i$-ésima variável; $\overline{T_{1}, T_{2}}, \overline{T_{3}}, \overline{R_{1}}, \bar{R}_{2}, \overline{R_{3}}$ $=$ médias gerais da transmitância $\left(\tau^{*}\right)$ e refletância $\left(\rho^{*}\right)$ efetivas do pelame, respectivamente, para as classes do comprimento de onda.
O método da Máxima Verossimilhança Restrita (Restricted Maximum Likelihood-REML) foi utilizado nas análises univariadas e bivariadas para estimar os componentes de variância, covariância e os parâmetros genéticos, sob um modelo animal, utilizando-se o sistema MTDFREML (Boldman et al., 1993). Nas análises, o critério de convergência considerado foi a variância do simplex menor que $10^{-9}$. Os valores iniciais das estimativas de variância foram obtidos por meio dos procedimentos de modelos mistos do SAS, tendo como base os modelos anteriormente citados considerando-se somente os efeitos fixos significativos e efeitos aleatórios.

Entretanto, foram considerados efeitos fixos e aleatórios significativos para IPP o ano do nascimento $(1, \ldots, 12)$; a estação do ano $(1, \ldots, 4)$; o número de inseminações $(1, \ldots, 11)$; pai $(1, \ldots, 199)$; a origem do pai da vaca $(1, \ldots, 3)$ e as regressões sobre a transmitância efetiva para os comprimentos de onda de 300-420 nm (1), 420-780 nm (2) e 780-850 nm (3). Para a variável IEP, foram considerados os efeitos fixos e aleatórios significativos: ano do primeiro parto $(1, \ldots, 10)$; número de inseminações $(1, \ldots, 17)$; efeito linear da idade da vaca; pai $(1, \ldots, 154)$; e as regressões sobre refletância efetiva para os comprimentos de onda de 300-420 nm (1), 420-780 nm (2) e 780-850 nm (3).As características do pelame foram determinadas utilizando-se os mesmos modelos analisados, os efeitos fixos e aleatórios significativos observados por Maia et al. (2003) para os mesmos animais e a base de dados do pelame deste estudo.

Em termos matriciais, os modelos (7) e (8) podem ser descritos como:

$\mathrm{Y}=\mathrm{X} b+\mathrm{Z} a+\mathrm{e}$

em que: $Y=$ vetor $n_{i} x 1$ de $n_{i}$ observações fenotípicas de $\mathrm{i}=$ IPP e IEP; $\mathrm{n}_{\mathrm{i}}=$ número de observações nos indivíduos; $\mathrm{X}=$ matriz $\mathrm{n}_{\mathrm{i}} \mathrm{xp}$ de incidência de $\mathrm{p}$ níveis dos efeitos fixos; $\mathrm{b}=$ vetor $\mathrm{px} 1$ de efeitos fixos e covariáveis; $\mathrm{Z}=$ matriz bloco diagonal $n_{\mathrm{i}} \mathrm{xn}_{\mathrm{i}}$ de incidência dos valores genéticos, de ordem nxq, para efeitos aleatórios do animal; $\mathrm{a}=\mathrm{qx} 1$ vetor de efeitos aleatórios do animal; e e = vetor $\mathrm{nx} 1$ dos efeitos aleatórios do erro. Outras definições: $q=$ número de níveis de efeitos aleatórios; $p$ = número de níveis de efeitos fixos e covariáveis.

As pressuposições adotadas no modelo (10) foram $E(\mathrm{y})=\mathrm{Xb} ; E(\mathrm{a})=E(\mathrm{e})=0 ;$ em que o efeito do erro, que inclui os efeitos genético ambiental e genético não-aditivo, é distribuído independentemente com $\sigma^{2}$. Então, $\operatorname{var}(\mathrm{e})=$ I $\sigma_{\mathrm{e}}^{2}=\mathrm{R} ; \operatorname{var}(\mathrm{a})=\mathrm{A} \sigma^{2} \mathrm{~A}_{\mathrm{a}} \mathrm{e} \operatorname{cov}(\mathrm{a}, \mathrm{e})=\operatorname{cov}(\mathrm{e}, \mathrm{a})=0$, em que A é o numerador da matriz de parentesco dos indivíduos; $\mathrm{I}=$ matriz identidade; $\sigma_{\mathrm{a}}^{2}=$ variância aditiva direta das características; $\sigma^{2}{ }_{\mathrm{e}}=$ variância residual das características. 
Desde que $\operatorname{cov}(\mathrm{a}, \mathrm{e})=\operatorname{cov}(\mathrm{e}, \mathrm{a})=0$, então, $\operatorname{var}(\mathrm{y})=\mathrm{ZAZ}{ }^{\prime} \sigma^{2}{ }_{\mathrm{a}}+\mathrm{R}$.

O modelo misto de equações para o modelo (10) foi:

$\left[\begin{array}{ll}\mathrm{X}^{\prime} \mathrm{X} & \mathrm{X}^{\prime} \mathrm{Z} \\ \mathrm{ZX} & \mathrm{Z}^{\prime} \mathrm{Z}+\mathrm{A}_{\alpha}^{-1}\end{array}\right]\left[\begin{array}{l}\hat{\mathrm{b}} \\ \hat{\mathrm{a}}\end{array}\right]=\left[\begin{array}{l}X^{\prime} Y \\ Z^{\prime} Y\end{array}\right]$

em que a $=\sigma^{2}{ } / \sigma^{2}$, ou $\left(1-h^{2}\right) / h^{2}$. Os demais termos foram definidos anteriormente.

As estimativas da herdabilidade direta e das correlações genéticas, fenotípicas e ambientais foram calculadas como:

Herdabilidade $=\hat{h}^{2}=\left(\hat{\sigma}^{2} a / \hat{\sigma}_{f}{ }_{f}\right)$

Correlação genética $=\hat{r}_{a}=\hat{\sigma}_{a a^{\prime}} / \sqrt{\hat{\sigma}_{a}^{2}} \hat{\sigma}_{a^{\prime}}^{2}$

Correlação fenotípica $=\hat{r}_{f^{\prime}}=\hat{\sigma}_{f f^{\prime}} / \sqrt{\hat{\sigma}_{f}^{2}} \hat{\sigma}_{f^{\prime}}^{2}$

Correlação ambiental $=\hat{r}_{e}=\hat{\sigma}_{e e^{\prime}} / \sqrt{\hat{\sigma}_{e}^{2}} \hat{\sigma}_{e^{\prime}}^{2}$

em que $\hat{\sigma}_{f}{ }^{2}=\hat{\sigma}_{a}^{2}+\hat{\sigma}_{e}^{2} ; \hat{\sigma}_{f f^{\prime}}{ }^{2}=\hat{\sigma}_{a d^{\prime}}{ }^{2}+\hat{\sigma}_{e \dot{e}}^{2} ; \hat{\sigma}_{a d}^{2}=$ covariância genética entre as características; $\hat{\sigma}_{e e^{\prime}}^{2}=$ covariância residual entre as características.

\section{Resultados e Discussão}

As médias gerais e os erros-padrão para as características morfológicas $\left(\mathrm{P}, \mathrm{E}, \mathrm{C}, \mathrm{N}\right.$ e D) e radiativas $\left(\tau^{*}{ }_{1,2 \text { e } 3} \mathrm{e}\right.$ $\rho^{*}{ }_{1,2}$ e 3 ) do pelame e para o desempenho reprodutivo (IPP e IEP) das vacas holandesas são apresentados na Tabela 1.
A média geral \pm erro-padrão da idade ao primeiro parto foi de $792 \pm$ 2,67 dias. Becerril et al. (1993), na Flórida, Estados Unidos, obtiveram resultado semelhante (799 \pm 89 dias). Grosshans et al. (1997) observaram idade ao primeiro parto de $731 \pm 23$ dias para a raça holandesa na Nova Zelândia dois meses menor que a observada neste estudo. Para o intervalo de partos, obteve-se neste estudo valor de 415 $\pm 4,59$ dias, próximo ao observado por Zambianchi et al. (1999), de 422 \pm 93,5 dias, em vacas holandesas no Brasil. Becerril et al. (1993) observaram intervalo menor (406 dias), na Flórida, assim como Silva et al. (1998) e Ojango \& Pollott (2001) obtiveram emseus estudos intervalos de partos menores (401 e 406 dias em vacas holandesas no Brasil e no Kênia, respectivamente).

Como característica morfológica do pelame, os animais apresentaram, de modo geral, maior proporção de área de malhas negras em relação à superfície corporal $(71,67 \%)$, capa pouco espessa $(2,64 \mathrm{~mm})$, pouco densa (1050 pêlos/ $\left.\mathrm{cm}^{2}\right)$, com pêlos curtos $(12,81 \mathrm{~mm})$ e grossos $(62,98 \mu \mathrm{m})$, indicando boa adequação do pelame às condições de ambiente tropical. Estes resultados comprovaram diferenças claras, quando comparados aos observados por Udo (1978), em vacas holandesas na Holanda (24,8 e 25,6 mm de comprimento para os pelames preto e branco, respectivamente); 39,3 e 37,7 mm de diâmetro e 1.465 e 1.430 pêlos $/ \mathrm{cm}^{2}$. De acordo com Maia et al. (2003), as condições naturais do ambiente tropical às quais os animais foram submetidos no Brasil, certamente, foram a causa dessas modificações.

Tabela 1 - Média geral, erro-padrão e número de observações para características morfológicas e radiativas do pelame e desempenho reprodutivo de vacas holandesas

Table 1 - Overall mean, standard error (SE) and number of records for reproductive and hair coat traits of Holstein cows

\begin{tabular}{|c|c|c|c|c|}
\hline \multirow[t]{2}{*}{$\begin{array}{l}\text { Característica } \\
\text { Trait }^{a}\end{array}$} & \multicolumn{2}{|c|}{$\begin{array}{c}\text { Idade ao primeiro parto } \\
\text { Age at first calving }\end{array}$} & \multicolumn{2}{|c|}{$\begin{array}{l}\text { Intervalo de partos } \\
\text { Calving interval }\end{array}$} \\
\hline & $\mathrm{N}$ & $\begin{array}{c}\text { Média geral } \pm \text { erro-padrão } \\
\text { Overall mean } \pm S E\end{array}$ & $\mathrm{n}$ & $\begin{array}{c}\text { Média geral } \pm \text { erro-padrão } \\
\text { Overall mean } \pm S E\end{array}$ \\
\hline $\mathrm{P}$ & 919 & $71,677 \pm 0,942$ & 595 & $70,440 \pm 1,192$ \\
\hline $\mathrm{E}$ & 919 & $2,647 \pm 0,017$ & 595 & $2,660 \pm 0,022$ \\
\hline $\mathrm{C}$ & 919 & $12,819 \pm 0,112$ & 595 & $12,694 \pm 0,141$ \\
\hline $\mathrm{N}$ & 919 & $1.050,650 \pm 13,002$ & 595 & $989,422 \pm 15,452$ \\
\hline $\mathrm{D}$ & 919 & $62,982 \pm 0,183$ & 595 & $62,646 \pm 0,229$ \\
\hline $\mathrm{t}^{*}$ & 919 & $0,005 \pm 0,002$ & 595 & $0,007 \pm 0,003$ \\
\hline$t^{*}{ }_{2}^{1}$ & 919 & $0,008 \pm 0,003$ & 595 & $0,011 \pm 0,005$ \\
\hline$t^{* 2} 3$ & 919 & $0,011 \pm 0,004$ & 595 & $0,014 \pm 0,006$ \\
\hline $\mathrm{r}_{*}^{*} 1$ & 919 & $0,062 \pm 0,001$ & 595 & $0,063 \pm 0,001$ \\
\hline $\mathrm{r}^{* 1}$ & 919 & $0,155 \pm 0,003$ & 595 & $0,157 \pm 0,004$ \\
\hline$r_{3}^{* 2}$ & 919 & $0,191 \pm 0,004$ & 595 & $0,194 \pm 0,005$ \\
\hline IPP & 919 & $792,522 \pm 2,677$ & - & - \\
\hline IEP & - & - & 595 & $415,109 \pm 4,593$ \\
\hline
\end{tabular}

a Proporção de área de malhas negras em relação à superfície corporal ( $P, \%$; espessura da capa de pelame ( $E$, mm) comprimento médio dos pêlos ( $C$, $\mathrm{mm})$; número de pêlos por unidade de área da epiderme $\left(\mathrm{N}\right.$, pêlos $\left./ \mathrm{cm}^{2}\right)$; diâmetro médio dos pêlos $(\mathrm{D}, \mu \mathrm{m})$; transmitância efetiva $\left(\tau^{*}\right)$ refletância efetiva $\left(\rho^{*}\right)$ do pelame para cada comprimento de onda: $300-420 \mathrm{~nm}(1), 420-780 \mathrm{~nm}(2)$ e $780-850 \mathrm{~nm}(3)$. As características do pelame, exceto para $(P)$, foram consideradas como média do pelame branco e preto; idade ao primeiro parto (IPP, dias); intervalo de partos (IEP, dias).

a Percentage of black meshes $(P, \%)$; hair coat thickness $(E, m m)$; hair length $(C, m m)$; number of hair per unit area $(N$, hair/cn? $)$; hair diameter $(D, \mu m)$; effective transmission $\left(\tau{ }^{*}\right)$ and reflection $\left(\rho^{*}\right)$ for each wavelength: $300-420 \mathrm{~nm}(1), 420-780 \mathrm{~nm}$ (2) and $780-850 \mathrm{~nm}$ (3); The hair coat characteristics were averages of black and white meshes, except for percentage of black meshes. Age at first calving (IPP, days); calving interval (IEP, days). 
Neste estudo, os valores de transmitância efetiva variaram de 0,005 a 0,011 e os de refletância efetiva de 0,062 a 0,191 para cada comprimento de onda. De acordo com Silva et al. (2003), as diferenças na refletância do pelame e da epiderme e na absortância do pelame podem ser negligenciadas no caso de animais demesma raça e pigmentação da epiderme, o que significa que a pequena variação da refletância não depende das características morfológicas do pelame.

Por outro lado, segundo Maia et al. (2005), a transmitância efetiva depende totalmente das características morfológicas do pelame. Assim, é importante ressaltar que a pigmentação do pelame e da epiderme constitui proteção efetiva contra a radiação ultravioleta. Quanto maior a proporção de malhas negras, menor a penetração de radiação ultravioleta (menor que $300 \mathrm{~nm}$ ) em vacas holandesas, o que proporciona maior proteção à radiação solar de regiões tropicais (Silva, 1999; Silva et al., 2001). Como a cor negra absorve mais radiação térmica e reflete menos, o aquecimento do pelame é maior, porém, pode ser compensado por pêlos curtos, grossos e bem assentados, além de maior capacidade de sudação (Silva, 2000b).

Entretanto, uma vez que na raça Holandesa a pigmentação da epiderme acompanha a do pelame, Silva (1999) propôs duas alternativas: sob regime de manejo em pastagem, deve-se dar preferência a animais predominantemente negros, oferecendo-lhes sombra suficiente na pastagem; sob regime de estabulação, animais predominantemente brancos são mais vantajosos; em qualquer caso, a capa de pelame deve ser a menos espessa possível, com pelos curtos e grossos.

Os resultados da análise de variância da idade ao primeiro parto e do intervalo de partos são apresentados na Tabela 2. Variações significativas $(\mathrm{P}<0,05)$ da idade ao primeiro parto foram observadas para os efeitos de ano do nascimento, de número de inseminações por concepção, de pai dentro de origem e de transmitâncias efetivas da radiação solar para os seguintes comprimentos de onda: $300-420 \mathrm{~nm}$ (1), 420-780 nm (2) e 780-850 nm (3).

$\mathrm{O}$ efeito de maior impacto na variação da idade ao primeiro parto foi o número de inseminações (quadrado mínimo $=165672,67 ; \mathrm{P}<0,001)$. Novilhas inseminadas uma única vez diferiram significativamente daquelas com mais de uma inseminação por concepção. Alterações na duração e no comportamento sexual durante o estro, no aumento da porcentagem de oócitos anormais e na incidência de morte embrionária precoce e, conseqüentemente, de repetições de cios, são as manifestações mais comumente observadas

Tabela 2 - Resumo da análise de variância da idade ao primeiro parto e intervalo de partos em vacas Holandesas Table 2 - Summary of analyses of variance for age at first calving and calving interval of Holstein cows

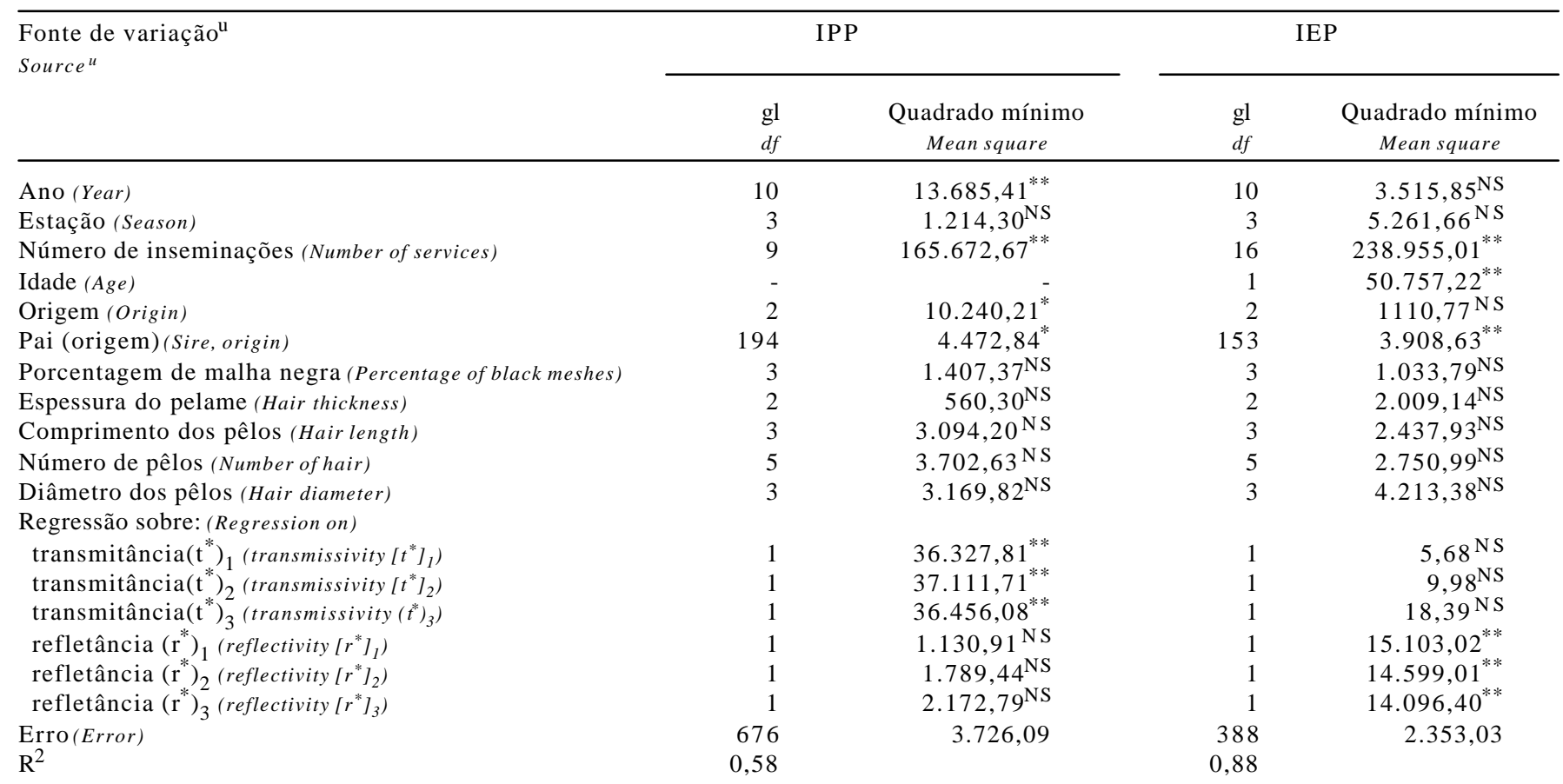

U unidades: idade ao primeiro parto (IPP); intervalo de partos (IEP); transmitância efetiva $\left(\tau^{*}\right)$ e refletância efetiva $\left(\rho^{*}\right)$ do pelame para cada comprimento de onda: $300-420 \mathrm{~nm}(1), 420-780 \mathrm{~nm}(2)$ e $780-850 \mathrm{~nm}(3) ;{ }^{* \star} \mathrm{P}<0,01 ;{ }^{*} \mathrm{P}<0,05$; NS $\mathrm{P}>0,05$

${ }^{U}$ units: Age at first calving, (IPP); calving interval (IEP); effective transmission $\left(\tau^{*}\right)$ and reflection $\left(\rho^{*}\right)$ for each wavelength: $1300-420 \mathrm{~nm}(1), 420-780 \mathrm{~nm}(2)$ e $780-850 \mathrm{~nm}(3) ;{ }^{* *} P<0.01 ;{ }^{*} P<0.05$; NS $P>0.05$ 
durante o verão, em decorrência do estresse térmico (Wolfenson et al., 2000). Entretanto, a estação do ano não teve efeito significativo $(\mathrm{P}>0,05)$ sobre a idade ao primeiro parto neste estudo. Diferentemente, a baixa fertilidade em estações quentes do ano foi reportada por Hansen (1997) e Wolfenson et al. (2000).

$\mathrm{O}$ efeito de pai dentro de origem foi marcante $(\mathrm{P}<0,01)$ sobre a precocidade das novilhas, implicando na importância da escolha do touro com mérito para precocidade reprodutiva de suas filhas. Segundo Schillo et al. (1992), a genética do pai e a nutrição assumem grande importância quanto à idade da novilha à concepção.

As transmitâncias efetivas do pelame para cada comprimento de onda foram, entre as características do pelame analisadas, as únicas variáveis significativas $(\mathrm{P}<0,01)$ sobre a variação da idade ao primeiro parto. A transmitância é a taxa de transferência de energia radiante através do pelame por unidade de profundidade $\left(\mathrm{Wm}^{-1}\right)$, como definido por Silva (2000b). Portanto, quanto maior a transmitância maior a perda de calor por radiação, observando-se que esta transmitância depende totalmente das características morfológicas do pelame (Maia et al., 2005).

$\mathrm{Na}$ análise de variância do intervalo de partos (Tabela 2), variações significativas $(\mathrm{P}<0,01)$ foram observadas para os efeitos de número de inseminações por concepção, idade, pai dentro da origem e para as refletâncias efetivas do pelame.

Vacas mais velhas tiveram intervalos maiores que as jovens. Resultado semelhante foi observado por McGregor \& Casey (1999), em que o efeito da idade foi significativo sobre o intervalo de partos, visto que animais mais velhos tiveram intervalos maiores. Assim como para a variável idade ao primeiro parto, o efeito de pai dentro de origem também foi uma importante fonte de variação do intervalo de partos. Ojango \& Pollott (2001) também observaram valor genético superior para touros da raça Holandesa de origem americana ( $-0,83$ dias) ou keniana ( $-0,63$ dias $)$, em comparação aos canadenses (-1,31 dias), para o intervalo de partos de suas filhas, no Kênia. A refletância foi significativa $(\mathrm{P}<0,01)$ na variação do intervalode partos, indicando grande importância da reflexão de radiação solar, apesar de os animais serem manejados à sombra, o que comprova a importância da radiação solar em ambiente tropical.

Estimativas de variância fenotípica, ambiental, genética e herdabilidade das características do pelame e das variáveis reprodutivas analisadas são apresentadas na Tabela 3 .

As variâncias genéticas aditivas das variáveis reprodutivas foram relativamente baixas, especialmente para o intervalo de partos. A estimativa da herdabilidade direta obtida para a idade ao primeiro parto $(0,23 \pm 0,079)$ sugere que esta característica apresentou variação genética aditiva suficiente para responder à seleção.

De acordo com Becerril et al. (1993), a estimativa de herdabilidade da idade ao primeiro parto incluindo a cor do pelame no modelo foi de $0,19 \pm 0,10$, inferior ao valor observado neste resultado. Silva et al. (1998) estimaram valor inferior $(0,05 \pm 0,01)$ em análise conjunta com a produção de leite, em vacas holandesas, considerando dados do período de 1980 a 1992 no Brasil. Miranda (1988), em revisão, mencionou valores superiores (entre 0,49 e 0,59 ) para esta variável em vacas holandesas no Brasil.

A herdabilidade do intervalo de partos $(0,19 \pm 0,099)$ neste estudo foi superior às encontradas na literatura. Resultados inferiores foram observados por Miranda (1988), de 0 a 0,08; Silva (1998), de 0,12 $\pm 0,02$; Zambianchi et al.

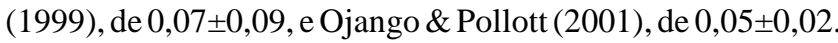
As estimativas de herdabilidade de variáveis de desempenho

Tabela 3 - Estimativas de variância fenotípica $\left(\sigma_{\mathrm{p}}{ }^{2}\right)$, ambiental $\left(\sigma_{\mathrm{e}}{ }^{2}\right)$ e genética $\left(\sigma_{\mathrm{g}}{ }^{2}\right)$ e herdabilidade $\left(\mathrm{h}^{2}\right)$ das características morfológicas do pelame e do desempenho reprodutivo de vacas holandesas em ambiente tropical

Table 3 - Phenotypic $\left(\sigma_{p}^{2}\right)$, environmental $\left(\sigma_{e}^{2}\right)$ and genetic $\left(\sigma_{g}{ }^{2}\right)$ variances and heritability $\left(h^{2}\right)$ estimates for reproductive and hair coat traits of Holstein cows in tropical environment

\begin{tabular}{|c|c|c|c|c|c|}
\hline \multirow[t]{2}{*}{$\begin{array}{l}\text { Característica } \\
\text { Trait }^{A}\end{array}$} & \multirow[t]{2}{*}{ (n) } & \multicolumn{4}{|c|}{$\begin{array}{l}\text { Parâmetro } \\
\text { Parameter }\end{array}$} \\
\hline & & $\sigma_{p}^{2}$ & $\sigma_{\mathrm{e}}^{2}$ & $\sigma_{\mathrm{g}}^{2}$ & $\mathrm{~h}^{2}$ \\
\hline IPP & 918 & $3.868,580$ & $2.992,865$ & 875,715 & $0,23 \pm 0,079$ \\
\hline IEP & 595 & $2.825,417$ & $2.402,498$ & 422,918 & $0,19 \pm 0,099$ \\
\hline $\mathrm{E}$ & 918 & 0,127 & 0,122 & 0,0055 & $0,04 \pm 0,050$ \\
\hline $\mathrm{C}$ & 918 & 8,090 & 5,166 & 2,924 & $0,36 \pm 0,090$ \\
\hline $\mathrm{D}$ & 918 & 27,374 & 10,890 & 17,280 & $0,63 \pm 0,080$ \\
\hline $\mathrm{P}$ & 918 & 774,17 & 191,970 & 582,200 & $0,75 \pm 0,080$ \\
\hline
\end{tabular}

A Idade ao primeiro parto (IPP); intervalo de partos (IEP); espessura da capa de pelame (E); comprimento médio dos pêlos (C); número de pêlos por área $(\mathrm{N})$; diâmetro médio dos pêlos $(\mathrm{D})$; porcentagem de malha negra $(P)$.

${ }^{\mathrm{A}}$ Age at first calving, (IPP); calving interval (IEP); coat thickness (E), hair length (C); number of hair per unit area (N); hair diameter (D); Percentage of black meshes (P). 
reprodutivo são geralmente baixas e menores que 0,15 (Dong \& Van Vleck, 1989). A variação genética aditiva foi pouco expressiva, indicando maior impacto das variações em decorrência de fatores ambientais e da atuação de genes dominantes ou epistáticos.

Destacaram-se os altos valores de herdabilidade para a porcentagem de malhas negras $\left(h^{2}=0,75 \pm 0,08\right)$ e o diâmetro médio dos pêlos $\left(h^{2}=0,63 \pm 0,08\right)$. Becerril et al. (1993) e Reinsch et al. (1999) obtiveram altos valores de herdabilidade para proporção de malha negra na raça Holandesa $(0,71 \pm 0,03 ; 0,88 \pm 0,08$, respectivamente). A seleção em ambiente tropical poderia ser facilmente direcionada para vacas predominantemente pretas, considerando o alto valor de $h^{2}$. Esses animais são mais protegidos contra a radiação solar por apresentarem epiderme altamente pigmentada, embora com maior absorção de energia térmica (Silva et al., 2001; Maia et al., 2003). Entretanto, a seleção deve ser realizada para se obterem animais com pelame menos denso e pêlos curtos e grossos para favorecer as perdas de calor latente e sensível.

O comprimento médio dos pêlos apresentou herdabilidade igual a $0,36 \pm 0,09$, superior à encontrada por Silva et al. (1988), de 0,08 $\pm 0,07$, e semelhante à descrita por Maia et al. (2003), de 0,37 $\pm 0,07$, para o pelame preto. A espessura do pelame e o número de pêlos apresentaram valores de $h^{2}$ iguais a 0,04 e 0,05 , respectivamente, superiores aos reportados por Maia et al. (2003), de 0,08 e 0,11, para essas mesmas características no pelame preto. Esses autore $\mathrm{s}$ mostraram que, de acordo com os coeficientes de correlação genética entre as características do pelame, a seleção para diminuição do comprimento resultaria em uma seleção simultânea para menor espessura e menor número de pêlos, o que seria desejável em ambiente tropical.

As estimativas das correlações genéticas, fenotípicas e ambientais entre as características do pelame, idade ao primeiro parto e intervalo de partos são apresentadas na Tabela 4. Uma vez que não foram encontradas outras referências sobre as correlações entre variáveis reprodutivas e adaptativas do pelame de bovinos, os resultados encontrados não puderam ser comparados.

Os valores das correlações genéticas entre as variáveis reprodutivas e adaptativas do pelame para espessura (exceto entre IEP) e entre número e diâmetro foram superiores aos das correlações fenotípicas. De modo geral, as estimativas de correlações fenotípicas foram muito baixas e não-significativas, à exceção da correlação fenotípica entre IPP e número de pêlos, que apresentou significância $(-0,08 ; \mathrm{P}<0,01)$.

A respeito das estimativas das correlações genéticas entre IPP, IEP e características do pelame, vale destacar as associações entre diâmetro médio dos pêlos e IPP $(-0,37 \pm 0,17)$ e IEP $(-0,49 \pm 0,27)$, que foram negativas (favoráveis), medianas e significativas. Estes resultados indicaram que a seleção praticada para maior diâmetro dos pêlos, o que constituiria a melhor escolha de tipo de pelame para animais em ambiente tropical, resultaria em seleção simultânea para precocidade sexual e menor intervalo de partos. É razoável esperar que vacas com pêlos mais grossos tenham maior capacidade de perder o excesso de calor corporal, por condução e convecção, para a camada de ar externa, o que, conseqüentemente, diminui os efeitos deletérios do estresse térmico sobre o desempenho reprodutivo.

A espessura da capa de pelame e o número de pêlos apresentaram valores estimados de correlações genéticas entre IPP medianos a alto e negativos $(-0,56 \pm 0,46$ e $-0,66 \pm 0,43$; respectivamente), mas foram desfavoráveis. Estes resultados sugeriram que a seleção de animais para menor espessura da capa de pelame e para menor número de pêlos, ou seja, paramaior tolerância ao calor, resultaria em seleção simultânea para maior idade ao primeiro parto, o que seria indesejável. Em contraste, as estimativas de

Tabela 4- Correlações genéticas, fenotípicas e ambientais entre características do pelame, idade ao primeiro parto e intervalo de partos de vacas holandesas em ambiente tropical

Table 4 - Genetic, phenotypic and environmental correlation estimates of hair coat traits, age at first calving and calving interval of Holstein cows in tropical environment

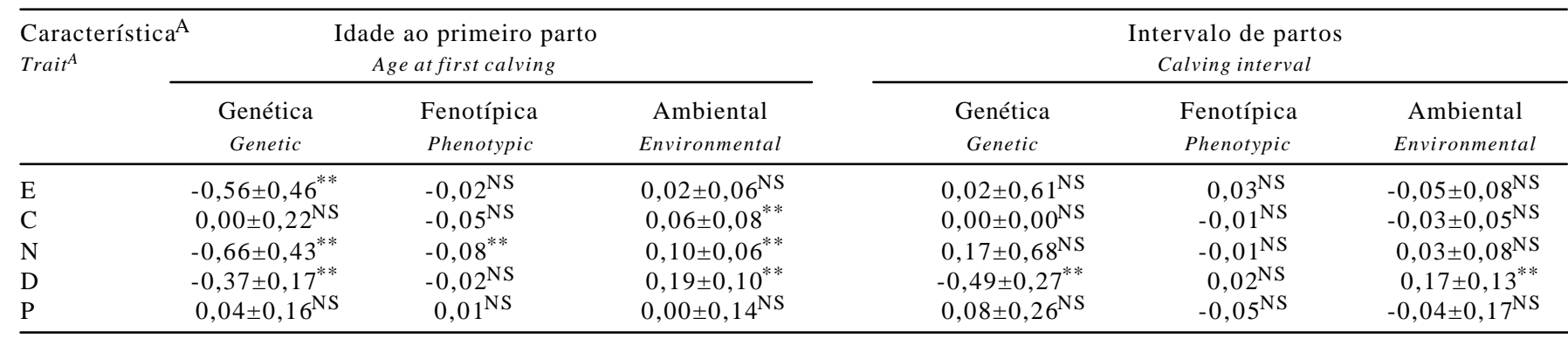

A Espessura da capa de pelame $(E)$; comprimento médio dos pêlos $(C)$; número de pêlos por unidade de área (N); diâmetro médio dos pêlos (D); porcentagem de malha negra $(P)$.

${ }^{A}$ Coat thickness $(E)$, hair length $(C)$; number of hair per unit area $(N)$; hair diameter $(D)$; percentage of black meshes $(P)^{* *} \mathrm{P}<0.01$; ${ }^{N S} \mathrm{P}>0.05$. 
correlação genética entre as variáveis reprodutivas (IPP e IEP) e o comprimento médio dos pêlos foram nulas. Este resultado comprovou independência dos genes responsáveis pela herança genética aditiva de IPP, IEP e comprimento médio dos pêlos.

É interessante destacar que as estimativas de correlações genéticas entre IEP e espessura e entre número de pêlos $(0,02 \pm 0,61$ e $0,17 \pm 0,68$; respectivamente) foram não-significativas. Portanto, a seleção para menor espessura da capa do pelame e menor número de pêlos não resultaria em melhor desempenho reprodutivo, em razão da independência destas características.

A proporção de malhas negras apresentou correlações genéticas próximas de zero e positivas entre $\operatorname{IPP}(0,04 \pm 0,16)$ e IEP $(0,08 \pm 0,26)$, indicando que, apesar de a estimativa ser de baixa magnitude, a seleção para animais com menor proporção de malhas negras resultaria em animais sexualmente mais precoces e com menores intervalos de partos. Todavia, a seleção para maior tolerância ao calor em ambiente tropical deve ser direcionada para animais predominantemente pretos, pois a pigmentação constitui uma proteção efetiva contra a radiação ultravioleta direta (Silva, 1999; Silva et al., 2001). Além disso, segundo Maia et al. (2003), pelames de cor preta, na raça Holandesa, geralmente são menos densos, com pêlos mais grossos e mais curtos, e estas características em conjunto melhoram a tolerância ao calor de animais da raça Holandesa manejados em ambientes quentes e úmidos.

\section{Conclusões}

A seleção para maior diâmetro dos pêlos resultaria em fêmeas sexualmente mais precoces. Entretanto, a seleção para menor espessura, menor comprimento e menor número de pêlos, bem como para maior porcentagem de malhas negras, não traria melhoramento genético ao desempenho reprodutivo dos animais.

Os resultados obtidos referem-se a um único rebanho de vacas holandesas manejadas à sombra em sistema livre de estabulação, com ventiladores e aspersores, nutrição e sanidade otimizadas. Neste contexto, a associação entre o tipo de pelame e o desempenho reprodutivo gera características de grande interesse científico e prático, principalmente para animais desta raça em ambientes tropicais, manejados em pastagens expostos ao sol.

\section{Literatura Citada}

BADINGA, L.; COLLIER, R.J.; THATCHER. W.W. et al. Effects of climatic and management factors on conception rate of dairy cattle in subtropical environment. Journal of Dairy Science, v.68, n.1, p.78-85, 1985.

BECERRIL, C.M.; WILCOX, C.J. Determination of percentage of coat color from registry certificates in Holsteins. Journal of Dairy Science, v.75, n.12, p.3582-3586, 1992.

BECERRIL, C.M.; WILCOX, C.J.; LAWLOR, T.J. et al. Effects of percentage of white coat color on Holstein cow production and reproduction in a subtropical environment. Journal of Dairy Science, v.74, n.8, p.2286-2291, 1993.

BOLDMAN, K.; KRIESE, L.; Van VLECK, L.D. A manual for use of MTDFREML: a set of programs to obtain estimates of variances and covariances (DRAFT). Lincoln: Department of Agriculture, Agriculture Research Service, 1993. 120p.

BOX, G.E.P.; COX, D.R. An analysis of transformations. Journal of Royal Statistical Society. Serie B, v.26, p.211-252, 1964

CAVESTANY, D.; El-WHISHY, A.B.; FOOT, R.H. Effect of season and fertility of Holstein cattle. Journal of Dairy Science v.68, n.6, p.1471-1478, 1985.

CENA, K.; MONTEITH, J.L. Transfer processes in animal coats. I. Radiative transfer. Procedure Royal Society London v.188, n.2, p.377-393, 1975.

DRAPPER, N.R.; SMITH, H. Applied regression analysis. 3.ed. New York: Wiley, 1998. 436p.

DONG, M.C.; Van VLECK, L.D. Correlations among first and second lactation milk yield and calving interval. Journal of Dairy Science, v.72, n.7, p.1933-1936, 1989.

GROSSHANS, T.; XU, Z.Z.; BURTON, L.J. et al.Performance and genetic parameters for fertility of seasonal dairy cows in New Zealand. Livestock Production Science, v.51, n.1, p.41-51, 1997

GWAZDAUSKAS, F.C.; WILCOX, C.J.; TATCHER, W.W. Environmental and managemental factors affecting conception rate in a subtropical climate. Journal of Dairy Science, v.58, n. 1, p. $88,1975$.

HANSEN, P.J. Effects of environment on bovine reproduction. In: YOUNGQUIST, R.S. (Eds.) Current therapy in large animal theriogenology. Philadelphia: W.B. Saunders, 1997. p.403-415.

LITTELL, R.C.; FREUND, R.J.; SPECTOR, P.SAS ${ }^{\circledR}$ System for linear models. 3.ed. Cary: 1991. 329p.

MAIA, A.S.C.; SILVA, R.G.; BERTIPAGLIA, E.C.A. Características do pelame de vacas Holandesas em um ambiente tropical: um estudo genético e adaptativo. Revista Brasileira de Zootecnia, v.32 n.4, p.843-853, 2003.

MAIA, A.S.C.; SILVA, R.G.; BERTIPAGLIA, E.C.A. Environmental and genetic variation of the effective radiative properties of the coat of Holstein cows under tropical conditions. Livestock Production Science, v.92, n.2, p.307-315, 2005.

MCGREGOR, R.G.; CASEY, N.H. Evaluation of calving interval and calving date as measure of reproductive performance in a beef herd. Livestock Production Science, v.57, n.2, p.181191, 1999.

MIRANDA, R.M. Estimativas de parâmetros genéticos de gado leiteiro no Brasil. Pesquisa Agropecuária Brasileira, v.23, n.3, p.317-328, 1988.

OJANGO, J.M.K.; POLLOTT, G.E. Genetic of milk yield and fertility in Holstein-Friesian cattle on large-scale Kenyan farms. Journal of Animal Science, v.79, n.7, p.1742-1750, 2001.

PUTNEY, D.J.; MULLINS, S.; THATCHER, W.W. et al. Embryonic development in superovulated dairy cattle exposed to elevated ambient temperatures between the onset of estrus and insemination. Animal Reproduction Science, v.19, n.1, p.37$51,1989$.

REINSCH, N.; THOMSEN, H.; XU, N. et al. A QTL for the degree of spotting in cattle shows synteny with the KIT locus on chromosome 6. Journal of Heredity, v.90, n.6, p.629-634, 1999.

RIVERA, R.M.; HANSEN, P.J. Development of cultured bovine embryos after exposure to high temperatures in the physiological range. Reproduction, v.121, n.1, p.107-115, 2001. 
SCHILLO, K.; HALL, J.B.; STANLEY, M.H. Effects of nutrition and season on the onset of puberty in beef heifer. Journal of Animal Science, v.70, n.12, p.3994-4005, 1992.

SILVA, M.V.G.B.; BERGMANN, J.A.G.; MARTINEZ, M.L. et al. Associação genética, fenotípica e de ambiente entre as medidas de eficiência reprodutiva e produção de leite na raça Holandesa. Revista Brasileira de Zootecnia, v.27, n.6, p.1115-1122, 1998.

SILVA. R.G.; ARANTES NETO, J.G.; HOLTZ -FILHO, S.V. Genetic aspects of the variation of the sweating rate and coat characteristics of Jersey cattle. Brazilian Journal Genetics, v.11, n.2, p.335-347, 1988.

SILVA, R.G. Estimativa do balanço térmico por radiação em vacas Holandesas expostas ao sol e à sombra em ambiente tropical. Revista Brasileira de Zootecnia, v.28, n.6, p.1403-1411, 1999.

SILVA, R.G. Introdução à bioclimatologia animal. São Paulo: Nobel, 2000a. 286p.

SILVA, R.G. Um modelo para a determinação do equilíbrio térmico de bovinos em ambientes tropicais. Revista Brasileira de Zootecnia, v.29, n.4, p.1244-1252, 2000b.

SILVA, R.G.; LA SCALA JR., N.; POCAY, P.L.B. Transmissão de radiação ultravioleta através do pelame e da epiderme de bovinos. Revista Brasileira de Zootecnia, v.30, n.6, p.19391947, 2001.
SILVA, R.G.; LA SCALA JR., N.; TONHATI, H. Radiative properties of the body surface of cattle and other animals. Transactions of ASAE, v.46, n.4, p.913-918, 2003.

THATCHER, W.W. Effects of seasons, climate and temperature on reproduction and lactation. Journal of Dairy Science, v. 57, n.12, p.360, 1974.

UDO, H.M.J. Hair coat characteristics in Friesian heifers in the Netherlands and Kenya. Wageningem: Meded. Landbouwhogeschool Veenman, 1978. 135p.

WOLFENSON, D.; ROTH, Z.; MEIDAN, R. Impaired reproduction in heat stressed: basic and applied aspects. Animal Reproduction Science, v.60, n.4, p.535-547, 2000.

ZAMBIANCHI, A.R.; FREITAS, M.A.R.; PEREIRA, C.S. Efeitos genéticos e de ambiente sobre produção de leite e intervalo de partos em rebanhos leiteiros monitorados por sistema de informação. Revista Brasileira de Zootecnia, v.28, n.6, p.1263-1267, 1999. 